\title{
The approach to steady state in microemulsions under shear flow
}

\author{
Domenico Suppa \\ Theoretical Physics, University of Oxford, \\ 1 Keble Road, OX1 3NP, U.K.
}

\begin{abstract}
We present an analitical study of the dynamical process of the approach to steady state for a driven diffusive system represented by the microemulsion phase of a ternary mixture. The external applied field is given by a "plane Couette" shear flow and the problem is studied within the framework of a time-dependent GinzburgLandau model. A Fokker-Planck equation for the probability distribution of the concentration fluctuations is developed in a self-consistent one-loop approximation and solved exactly, giving an analytical expression for the dynamic correlation functions of the system. For comparison to experimental work, we also show grey-scale plots of the scattering function at different times during the dynamical process for two different shear rates.
\end{abstract}

Key words: Non-equilibrium Statistical Mechanics, Complex Fluids

PACS: 61.20.Gy, 82.70.-y, 83.50.Ax

\section{Introduction}

Ternary mixtures made up of oil, water and surfactant agent are of considerable interest for both fundamental issues as well as for applications [1-3]. In these systems, three-phase coexistence is commonly observed between a waterrich phase, an oil-rich phase and a microemulsion. The latter is a mesoscopic structured phase where coherent domains of oil and water on scales between 100 and $1000 \AA$ form disordered isotropic intertwined structures on larger scales [4]. A very similar mesoscopic structure is observed in sponge phases in acqueous amphiphilic solutions [5,6] and in homopolymer blends containing small amounts of diblock copolymers which act as a surfactant [7-11].

Email address: suppa@thphys.ox.ac.uk (Domenico Suppa). 
After the earlier work by Gompper and Shick [12] in which they demonstrate that the failure of the microemulsion to wet the oil-water interface is a consequence of the microemulsion internal structure, various theoretical advances on similar ternary systems have been made [13-16]; in particular Kielhorn and Muthukumar [16] have investigated the weak segregation behaviour of a ternary mixture of $\mathrm{A}$ homopolymer, $\mathrm{B}$ homopolymer and $\mathrm{AB}$ diblock copolymer, showing several features which are identical to those seen in traditional oil/water/surfactant systems. These amphiphilic systems are now receiving renewed attention, since several light and neutron scattering experiments revealed the hallmarks of glassy dynamics, in the sense of large relaxation times, on their mesoscopic structures [17-22]. Other ways of establishing non-equilibrium states with slow dynamics are also possible. For instance, microemulsions can be driven to a slow out of equilibrium stationary regime by a weak shear. This perturbation accelerates the dynamics in such a way that the structural relaxation time decreases with increasing shear strength (a phenomenon usually known as shear-thinning). The shear flow can therefore be used as a probe of the nonequilibrium properties of these systems by adopting the shear rate $\dot{\gamma}$ as a control parameter [23]. Several scattering experiments have shown the very complex and diverse effects of shear flow on these systems after the steady-state has been reached [24-32]: nonlinear effects due to the flow give rise to strongly anisotropic suppression of the composition fluctuations; the phase diagram is affected by the flow which can induce order-disorder transitions; the rheological behaviour is highly non-Newtonian with significant intriguing shear-thinning and shear-thickening behaviour.

In the following, we shall find useful to refer to the concentration fluctuation (equal time) correlation function, calling it "structure factor", although such a name usually indicates the density fluctuation.

Theoretical efforts to understand these complex problems have also been made intensively. A mean field theory able to describe the scattering function of a diblock copolymer in the presence of a steady homogeneous flow field was developed by Fredrickson [33] as a generalization of the equilibrium theory of Leibler [34]. The theory predicts the wave vector dependence of the scattering intensity and the distribution function of composition fluctuations in the disordered phase. The concept of an increase of the order-disorder transition temperature due to the shear was first studied for small shear rates by Cates and Milner [35] who applied a self-consistent Hartree approximation to the nonequilibrated system and solved for the shear effect on this Hartree term. A more general self-consistent approach, not limited to small shear rates and with predictions which are in good agreement with experimental results [36-38], was developed by Huang and Muthukumar [39] who studied numerically the effect of shear rate on order-disorder and order-order transitions involving lamellar and cylindrical morphologies as well as the dependence of steady-state scattering function on shear. A one-loop self consistent Ginzburg-Landau model for describing the steady-state rheological behavior of oil/water/surfactant mix- 
tures under a plane Couette shear flow was considered by Pätzold and Dawson [40] who gave a numerical evaluation of the structure factor together with an analytical analysis in the limit of vanishing shear rate $\dot{\gamma}$. Their model was exactly solved in [41] for any value of $\dot{\gamma}$ showing that in a specific shear range the structure factor is characterized by four pronounced peaks in the plane of the shear and flow directions. This phenomenon, which was already predicted by Drolet et al. in a slightly different model [42] and also reported in scattering experiments on segregating mixtures [30,25], was interpreted in terms of a complex spatial pattern where interfaces with two different orientations coexist, with relative abundance tuned by $\dot{\gamma}$. On the other hand, the relaxation dynamics of microemulsions towards a steady state, which can be extremely slow, is also very intriguing: in fact, the absence of time translation invariance gives rise to a complex behaviour where different modes relax on different time scales and differently compete with the hydrodynamics time scales.

A systematic study of the dynamical behaviour of microemulsions and sponge phases in thermal equilibrium and in the presence of hydrodynamic modes has been developed by Hennes and Gompper in [43]: they use a field-theoretic perturbation theory which makes use of "response fields "[44-46] and whose key ingredient is a self-energy matrix having a block structure identical to the Gaussian correlation and response matrix. Within that framework, they were able to calculate the frequency-dependent (complex) viscosity, the sound velocity and damping in a one-loop approximation. However, the macroscopic relaxation of the system and the role of the different time scales that are involved are most clearly shown by the evolution of the scattering intensity and, in particular, its asymptotic behaviour for short and large times. This was calculated in [43] by using the first Born approximation for evaluating the correlation matrix and, as it was already pointed out by those authors, it is not clear to what extent such behaviour relies on the adopted approximation. As a result, a self-consistent calculation of the dynamic behaviour of microemulsions and sponge phases is expected to clarify this important point.

Of concern in this paper is the microemulsion phase only and its slow relaxation dynamics towards the steady state, associated with composition fluctuations under a plane Couette shear flow. We will focus on the time-dependent static structure factor (i.e. the average of the concentration fluctuation at equal time, whose time dependence comes from the transition from equilibrium to steady state), and perform a self-consistent one-loop calculation within the model previously adopted in [41], which has the significant feature that results can be formulated in terms of the physical correlation lengths, thereby making direct comparisons to experiment possible. The aim of the present work is to contribute in making a contact between the mesoscopic structural characteristic of microemulsions and the general macroscopic dynamic features of soft materials. The plan of this paper is as follows. In Sec. 2 we present the adopted Ginzburg-Landau model for the microemulsion and derive the Fokker-Planck 
equation for the probability distribution of composition fluctuations. In Sec. 3 we obtain its dynamic solution which gives an analytical expression for the time-dependent static structure factor. We discuss the scattering function convergence towards its steady-state value and show its morphologies during the relaxation process. In Sec. 4 we draw our conclusions.

\section{Ginzburg-Landau Model}

We shall consider a binary fluid of oil and water at 50:50 concentration, with the addition of non-ionic surfactant agent, in thermal equilibrium at a temperature $T>T_{c}$. In order to describe the ternary mixture, we adopt a GinzburgLandau model with a single scalar order parameter $\varphi$ representing the local concentration difference between oil and water components. The following coarse-grained Ginzburg-Landau free-energy, firstly proposed by Gompper and Schick [3], has been used extensively for investigations of the static structure and phase behavior of these systems $[47,3,12]$ :

$$
F[\varphi]=\int d^{3} x\left(f(\varphi)+\frac{1}{2} g(\varphi)|\vec{\nabla} \varphi|^{2}+\frac{c}{2}\left(\nabla^{2} \varphi\right)^{2}\right)
$$

where $f(\varphi)$ is a polynomial function of $\varphi$ which has three minima $\varphi_{1}<\varphi_{2}<$ $\varphi_{3}$, with $\varphi_{2}=0$, corresponding to the three homogeneous bulk phases, oilrich, microemulsion and water-rich respectively. Moreover $g(\varphi)=g_{0}+g_{2} \varphi^{2}$, with $g_{0}<0$ and $g_{2}>0$, in order to grasp the experimental evidence that the peak in the structure factor in the oil and water phases is at zero wavevector (so that $g(\varphi)$ must be sufficiently positive there), while the peak is at nonzero wavevector in the microemulsion (so that $g(\varphi)$ must be negative there). The last term, with a coefficient $c>0$, makes stable the free-energy at large momenta and weights the curvature of the interfaces where surfactant gathers. As we are concerned with the microemulsion phase, i.e. the disordered but structured region of the phase diagram where $a>0$ and a stability condition $4 a c-g_{0}^{2}>0$ is fulfilled, we will use a piecewise form of $f(\varphi)$ :

$$
f(\varphi)=\frac{a}{2} \varphi^{2}+\frac{b}{4 !} \varphi^{4}
$$

as proposed by Pätzold and Dawson [40], where the quartic term has been included in order to capture the fluctuation effects in the critical region. Although local concentration of surfactant does not appear in the free-energy, its average value is taken into account through the coefficients $g_{0}$ and $c$ : in particular the negative value of $g_{0}$, known as the surface tension parameter, allows us to model a characteristic feature of the microemulsion phase, i.e. 
the tendency of surfactant to create spontaneously interfaces. More refined models should include an additional order parameter to describe the density of surfactant (for a review about this point see [3]). In the following we will be concerned with the response of the system to a plane Couette shear flow where the average velocity field is expressed as

$$
\vec{v}(\vec{x})=\dot{\gamma} y \vec{e}_{x}
$$

where $\dot{\gamma}$ is the shear rate and has the dimensions of a frequency. The reference frame adopted is such that the $\mathrm{x}$-axis is along the flux, the $\mathrm{y}$-axis is along the velocity gradient and the z-axis is along the vorticity direction. $\vec{e}_{x}$ appearing in Eq. (3) is the unit vector in the flux direction. We will only consider shear flows such that the molecular relaxation times are less than the inverse of the shear rate $\dot{\gamma}$, i.e. the Deborah number $D=\dot{\gamma} \tau<<1$. Moreover, we will assume that, due to the highly viscous nature of the mixture, the fluctuations of the velocity field are small and that their coupling with the fluctuations of the order parameter is negligible [33,48]. Under these assumptions it is legitimate to apply the stochastic model given by Onuki and Kawasaki [49] and study in the momentum space the composition fluctuations by the following Langevinlike equation

$$
\frac{\partial}{\partial t} \varphi(\vec{k}, t)=\dot{\gamma} k_{x} \frac{\partial}{\partial k_{y}} \varphi(\vec{k}, t)-\Gamma k^{2}\left(\frac{\delta F}{\delta \varphi(-\vec{k}, t)}\right)+\eta(\vec{k}, t)
$$

where the Onsager coefficient $\Gamma$ is assumed to be a constant at a temperature $T$ sufficiently higher than $T_{c}$ (this assumtion cannot be extended to homopolymer mixtures where a k-dependent Onsager coefficient must be used [50]) and $\delta F / \delta \varphi$ is the usual thermodynamic force. $\eta$ is a stocastic white noise describing thermal fluctuations into the system: it is Gaussian distributed with mean zero and variance constructed to satisfy a (generalized) fluctuation-dissipation theorem [51]:

$$
\begin{aligned}
\langle\eta(\vec{k}, t)\rangle & =0 \\
\left\langle\eta(\vec{k}, t) \eta\left(\overrightarrow{k^{\prime}}, t^{\prime}\right)\right\rangle & =2 \Gamma k^{2} \delta\left(\vec{k}+\overrightarrow{k^{\prime}}\right) \delta\left(t-t^{\prime}\right)
\end{aligned}
$$

As usual, it is assumed that the fluctuating force is uncorrelated in time, which implies that one considers times much larger than the characteristic times during which internal modes equilibrate. Thereby, the stochastic process becomes Gaussian and Markovian [52]. In order to linearize the problem, we further assume that it is legitimate to approximate the term $\delta F[\varphi] / \delta \varphi(-\vec{k}, t)$ as $\chi(k) \varphi(\vec{k}, t)$, where $\chi^{-1}(k)$ can be regarded as a static susceptibility that depends on the correlation functions evaluated in the steady state. A self- 
consistent one-loop approximation gives

$$
\chi(k)=a^{R}+g^{R} k^{2}+c k^{4}
$$

with $a^{R}=a+b / 2 S_{0}^{\infty}+g_{2} S_{2}^{\infty}, g^{R}=g_{0}+g_{2} S_{0}^{\infty}$, and

$$
S_{n}^{\infty}=\lim _{t \rightarrow \infty} \int_{|\vec{k}|<\Lambda} \frac{d \vec{k}}{(2 \pi)^{3}}|\vec{k}|^{n} C(\vec{k}, t)
$$

In Eq. (8), $C(\vec{k}, t)=\langle\varphi(\vec{k}, t) \varphi(-\vec{k}, t)\rangle$ is the time-dependent static structure factor and $\Lambda$ is a cutoff proportional to the inverse of the length of the surfactant molecule. It is worth to note that, apart from the presence of renormalized coefficients, $\chi^{-1}(k)$ preserves the same functional expression of the equilibrium scattering function firstly deduced by Teubner and Strey in their seminal paper [47]. Such a form yields for $a>0, g_{0}<0$ and $c>0$ a single broad scattering peak at a non-zero wavevector, and a $k^{-4}$ decay at large $k$ which are properties experimentally observed for a variety of microemulsions containing comparable amounts of water and oil [53-56]. The present Hartree approximation has been used by Huang and Muthukumar (see [39] and references therein) for studying the steady-state structure factor in block copolymers. In the nonequilibrium problem in which we are interested in this paper, it will be used to study the dynamical process of the approach to the steady state. It represents the main simplification in our model and it is deemed to be adequate only close to the steady state (see also [57] and references therein, where a similar argument was used to study the approach to the steady state of staggered non-equilibrium particle systems and Ising chains). Therefore, the linearized Langevin equation for the compositions fluctuations is written as

$$
\frac{\partial}{\partial t} \varphi(\vec{k}, t)=\dot{\gamma} k_{x} \frac{\partial}{\partial k_{y}} \varphi(\vec{k}, t)-\Gamma k^{2} \chi(k) \varphi(\vec{k}, t)+\eta(\vec{k}, t)
$$

The set of Eqs. (5)-(9) is equivalent to the following Fokker-Planck equation for the probability distribution of the $\varphi(\vec{k}), P(\varphi(\vec{k}), t)[58]$ :

$$
\begin{aligned}
& \frac{\partial}{\partial t} P(\varphi(\vec{k}), t)=\int \frac{d \vec{k}}{(2 \pi)^{3}}\left\{\frac { \delta } { \delta \varphi ( \vec { k } ) } \left[-\dot{\gamma} k_{x} \frac{\partial \varphi(\vec{k})}{\partial k_{y}}+\right.\right. \\
& \left.\left.\quad+\Gamma|\vec{k}|^{2}\left(\chi(k) \varphi(\vec{k})+\frac{\delta}{\delta \varphi(-\vec{k})}\right)\right]\right\} P(\varphi(\vec{k}), t)
\end{aligned}
$$

Eq. (10) completely defines the stochastic relaxation process in which we are interested and represents the starting point for the analysis of the timedependent static structure factor that will be presented in the next session. 


\section{Results and Discussion}

Eq. (9) states a linear dependence of $\varphi(\vec{k}, t)$ from the noise $\eta(\vec{k}, t)$ : as the noise is Gaussian distributed, then the probability distribution $P[\varphi(\vec{k}, t)]$ must be Gaussian at each time. In fact the steady solution of Eq. (10), which represents the probability distribution for the field $\varphi$ in the steady state, is given by

$$
P_{0}\left(\varphi_{\vec{k}}\right)=\left(\frac{1}{\operatorname{det} C_{0}(\vec{k})}\right)^{1 / 2} \exp \left[-\frac{1}{2} \int \frac{d \vec{k}}{(2 \pi)^{3}} \frac{\varphi(\vec{k}) \varphi(-\vec{k})}{C_{0}(\vec{k})}\right]
$$

where $C_{0}$ stays for the steady-state structure factor and has the following expression [41]

$$
C_{0}(\vec{k})=\int_{0}^{\infty} d t e^{-\int_{0}^{t} \omega(k(\zeta)) d \zeta} \Gamma|\vec{k}(t)|^{2}
$$

where

$$
\vec{k}(t)=\left(k_{x}, k_{y}+\frac{\dot{\gamma} k_{x} t}{2}, k_{z}\right)
$$

and

$$
\omega(k)=\Gamma k^{2} \chi(k)
$$

is the thermal decaying rate, i.e. the rate at which order parameter fluctuations having a wavelength $\lambda=2 \pi / k$ relax at thermal equilibrium (in the absence of flow) [59,49]. Eqs. (7), (8) and (12) form a self-consistent relationship which accounts for the fluctuations under shear. By consideration of Eq. (12) it is apparent that for $\dot{\gamma}>>\omega(k)$ the composition fluctuations will be strongly distorted by the flow, i.e. the flux forces the $C_{0}(\vec{k})$ to be highly anisotropic. The integral in Eq. (12) is clearly positive, as it should be for a variance, and it is possible to verify that the solution (12) is unique: in fact every other solution different from that does not recover the Ornstein-Zernike form in the limit $\dot{\gamma} \rightarrow 0$ [49]. It is worth to note that $C_{0}(\vec{k})$ does not depend on the initial conditions. It will be shown in the following that the steady-state structure factor can be obtained from the time-dependent static one through a limit calculation $(t \rightarrow \infty)$ after which the correlation loses the memory of its initial value. Because of the Markovian character of the stochastic process and the translational invariance (see [49]), we make the following ansatz for the timedependent probability distribution of the field $\varphi$ during the dynamical process 
of the approach to steady state, that will be completely proved a posteriori:

$$
P(\varphi(\vec{k}), t)=G(t) \exp \left[-\frac{1}{2} \int \frac{d \vec{k}}{(2 \pi)^{3}} \frac{\varphi(\vec{k}) \varphi(-\vec{k})}{C(\vec{k}, t)}\right]
$$

where $G(t)=[1 /(\operatorname{det} C(\vec{k}, t))]^{1 / 2}$ is a normalization factor, and $C(\vec{k}, t)$ is the time-dependent static structure factor, which is given by the time-dependent solution of the following equation

$$
\frac{\partial C(\vec{k}, t)}{\partial t}=\dot{\gamma} k_{x} \frac{\partial C(\vec{k}, t)}{\partial k_{y}}-2 \omega(k) C(\vec{k}, t)+2 \Gamma k^{2}
$$

Eq. (16) can be obtained formally by taking the time derivative of $C(\vec{k}, t)=$ $\langle\varphi(\vec{k}, t) \varphi(-\vec{k}, t)\rangle$ and using the linearized Langevin Eq. (9), provided a regularization procedure is used for dealing with the difficulties coming from the fact that time differentiation and averaging do not commute. Such a regularization entails defining $\langle\varphi(\vec{k}, t) \eta(-\vec{k}, t)\rangle=\lim _{t^{\prime} \rightarrow t^{-}}\left\langle\varphi(\vec{k}, t) \eta\left(-\vec{k}, t^{\prime}\right)\right\rangle \theta\left(t-t^{\prime}\right)$, where the presence of the step function $\theta\left(t-t^{\prime}\right)$ reflects the causality of the Langevin equation, and then setting this equal to $\frac{1}{2}\langle\eta(\vec{k}, t) \eta(-\vec{k}, t)\rangle[60]$.

The first and second term in the R.H.S. of Eq. (16) are the convection term and the diffusion term respectively, while the third term is the fluctuation source. When the shear rate is large enough, the hydrodynamic force strongly interferes with the thermal decaying process and a nonlinear effect of suppression of the composition fluctuations takes place. We shall begin by solving the homogeneous problem:

$$
\begin{aligned}
\frac{\partial C(\vec{k}, t)}{\partial t}-\dot{\gamma} k_{x} \frac{\partial C(\vec{k}, t)}{\partial k_{y}} & =-2 \omega(k) C(\vec{k}, t) \\
C(\vec{k}, 0) & =C_{\text {init }}\left(k^{2}\right)
\end{aligned}
$$

where $C_{\text {init }}\left(k^{2}\right)=1 /\left(a+g_{0} k^{2}+c k^{4}\right)$ is the well known isotropic scattering function of the microemulsion at initial time, in the absence of flow [47,3], and its projection on each Cartesian plane is that of a circular volcano with radius proportional to the inverse correlation length of the system, as it is shown in Fig. (1).

The solution of the homogeneous problem is found by using the method of characteristics and it has the following form:

$$
C_{\text {hom }}(\vec{k}, t)=C_{\text {init }}\left(k^{2}(t)\right) \exp \left[-\int_{0}^{t} \omega\left(k\left(t-t^{\prime}\right)\right) d t^{\prime}\right]
$$




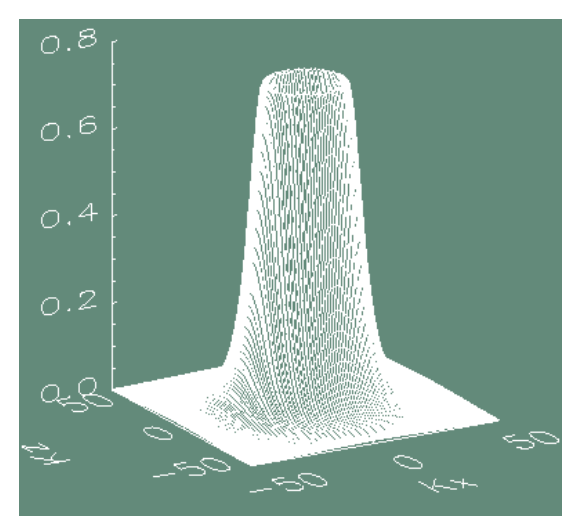

Fig. 1. The structure factor at initial time.

where $\omega(k)$ has been defined in Eq. (14). The full solution of Eq. (16) with the initial condition given in Eq. (18) is

$$
C(\vec{k} ; t)=C_{h o m}(\vec{k} ; t)+\int_{0}^{t} \Gamma|\vec{k}(m)|^{2} e^{-\int_{0}^{m} \omega(k(\zeta)) d \zeta} d m
$$

Taking the limit $t \rightarrow \infty$, it is straightforward to verify that $C(\vec{k}, t) \rightarrow C_{0}(\vec{k})$, i.e. the time-dependent field distribution $P(\varphi(\vec{k}), t)$ converges (in a weak sense) at infinite time towards the steady-state distribution $P_{0}(\varphi(\vec{k}))$ : this justifies a posteriori the previous ansatz (see Eq. (15)). Eq. (20) is the principal result of this paper: it represents an analytical expression for the timedependent static structure factor of microemulsions in the presence of a plane Couette shear flow. We shall now consider the behaviour of the structure factor for short times. In this limit we have

$$
\begin{aligned}
C(\vec{k} ; t) & =C_{h o m}(\vec{k} ; 0)+\Gamma k^{2}\left[1-C_{i n i t}\left(k^{2}(t)\right) \chi(k)\right] t+ \\
& +\frac{d C_{i n i t}\left(k^{2}\right)}{d k^{2}} \dot{\gamma} k_{x} k_{y} t+O\left(t^{2}\right)
\end{aligned}
$$

i.e. the scattering intensity grows linearly in t. A linear dependence on time t has been originally predicted by Hennes and Gompper in [43] within a different model. Nevertheless, the present self-consistent calculation differs from the former one in two important respects: firstly, the expression given in Eq. (21) shows a dependence on mode-coupling terms through $\chi(k)$ that does not appear in [43]: their presence is likely to be important in predicting the correct approach to the steady state. Secondly, the third term on the R.H.S. of Eq. (21) explicitly breaks the isotropy of the short time behaviour of the scattering function, unless $k_{x} k_{y}=0$. The significance of this anisotropic term is apparent in the following analysis and is amenable to experimental test. The predictions of our model are now investigated on the strongly structured microemulsion whose steady-state characteristic features have already 
been studied in [41] (to which we also refer for details on the specific values adopted for the system parameters). The role of the shear rate $\dot{\gamma}$ as a control parameter for probing the nonequilibrium properties of microemulsions has been already discussed in the Introduction. Therefore, we will now use two distinct values of the shear rate $(\dot{\gamma}=2$ and $\dot{\gamma}=8)$ which, even if both belonging to the region in which shear-thinning behaviour is observed, still produce very different mesoscopic structures into the system. The characteristic morphologies of the time-dependent static structure factor projections on both the planes $k_{z}=0$ and $k_{y}=0$, are shown in Fig. (2) (for $\dot{\gamma}=2$ ) and in Fig. (3) (for $\dot{\gamma}=8$ ) at four different times. The first time $(t=100)$ is chosen to

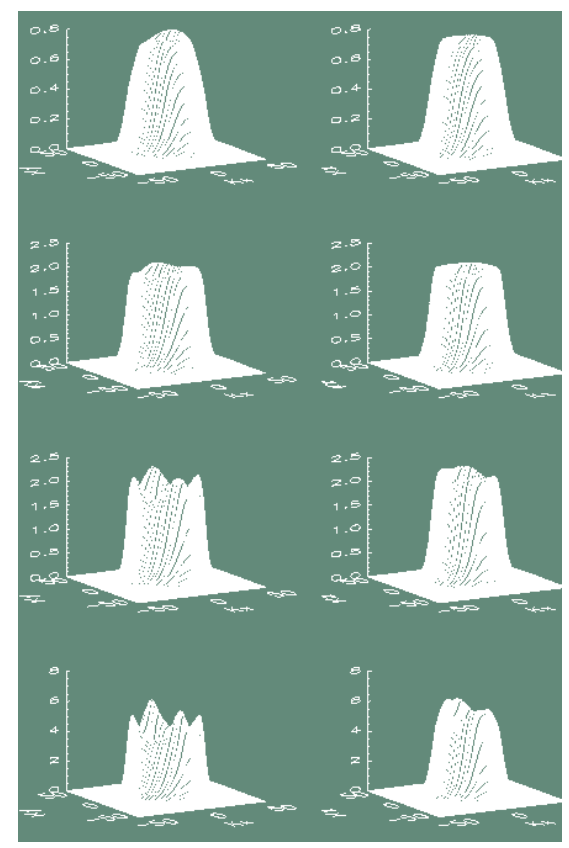

Fig. 2. Projections of the time-dependent static structure factor on the planes $k_{z}=0$ (left column) and $k_{y}=0$ (right column) at different times $\mathrm{t}=100,300,500$, 1000 (from top to bottom). The value of the shear rate is $\dot{\gamma}=2$.

be sufficiently small to observe the early deformations of the initial isotropic volcano shape, while the last one $(t=1000)$ is such that the steady-state morphologies previously found in [41] are recovered. The effect of the flow on the shape of the structure factor is to stretch it along a line oriented at $135^{\circ}$ to the $k_{x}$-axis and rotate it towards the $k_{y}$-axis $\left(k_{z}\right.$-axis) in the $k_{z}=0$ plane $\left(k_{y}=0\right.$ plane $)$. At any fixed time during the relaxation dynamics, the degree to which the scattering function is stretched and rotated depends on the value of $\dot{\gamma}$ and grows with it. It is worth to stress that the short-time morphologies in the $k_{y}=0$ plane are less anisotropic than those in the $k_{z}=0$ plane as a result of the absence of the term $\propto \dot{\gamma} k_{x} k_{y} t$ in Eq. (21) when $k_{y}=0$ : the predicted different degree of isotropy should be observable in scattering experiments and its possible confirmation would represent an important test of the present theory. Finally, the steady-state morphology of the structure factor strongly depends on $\dot{\gamma}$ : in the case $\dot{\gamma}=2$ the predicted four peaks structure 


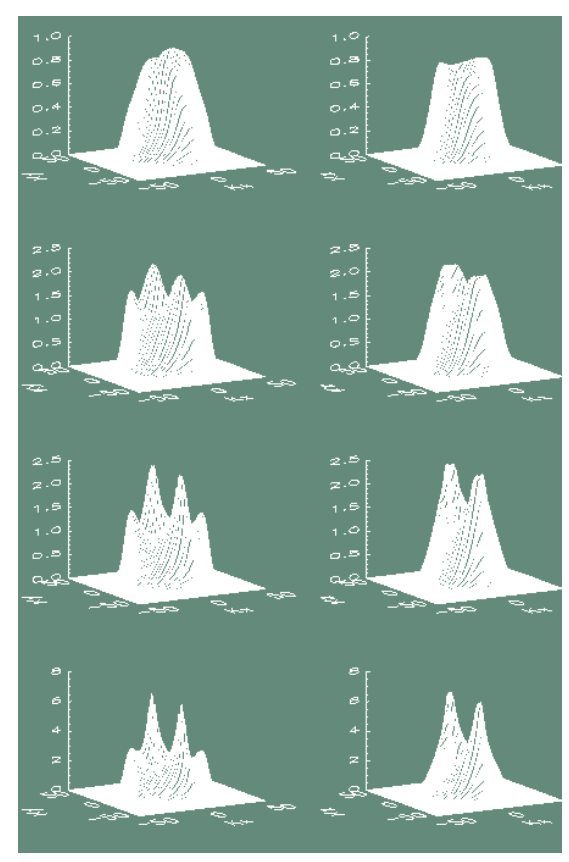

Fig. 3. Projections of the time-dependent static structure factor on the planes $k_{z}=0$ (left column) and $k_{y}=0$ (right column) at different times $\mathrm{t}=100,300,500$, 1000 (from top to bottom). The value of the shear rate is $\dot{\gamma}=8$.

can be related to a biphasic region with different allignment while above a certain shear rate the microemulsion is predicted to transform into a lamellar phase oriented along the flow, as it is observed for $\dot{\gamma}=8$.

\section{Conclusions}

In the present paper we have studied the response of microemulsion to the sudden application of a steady plane Couette shear flow. Analytical expressions for the dynamic scattering function and the distribution function of composition fluctuations have been developed and their convergence to steady-state values proven. The prediction of the model have been investigated on a structured microemulsion whose time-dependent static structure factor has been evaluated and explicitly shown at different times during the relaxation dynamics towards the steady state. The present results can be tested in neutron or Xray scattering experiments through which vital informations can be gained to further developing the analysis of the dynamical process. 


\section{Acknowledgments}

The author is grateful to R. Stinchcombe and L. Porcar for very helpful discussions and encouraging remarks. He also has a great pleasure in acknowledging the referee for making explicit the correct sense in which the name "structure factor" has been used through the paper.

\section{References}

[1] J. Meunier, D. Langevin and N. Boccara, Physics ofAmphiphilic Layers (Springer, Berlin, 1987).

[2] Micelles,Membranes,Microemulsions, and Monolayers, edited by W. Gelbart, D. Roux and A. Ben-Shaul (Springer, Berlin, 1995).

[3] G. Gompper and M. Schick in Phase Transition and Critical Phenomena, edited by C.Domb and J.Lebowitz (Academic,London,1994), Vol. 16, pp.1-176.

[4] P. G.de Gennes and C. Taupin, J.Phys.Chem. 86, 2294 (1982).

[5] G. Porte, J.Phys.Cond.Matt. 4, 8649 (1992).

[6] D. Roux, C. Coulon and M. Cates, J.Phys.Chem. 96, 4174 (1992).

[7] K. Binder, Adv.in Polym.Sci. 112, 181-299 (1994).

[8] M. Kosinas, J.Chem.Phys. 104, 405 (1995).

[9] D.G. Bucknall, J.S. Higgins and J. Penfold, Physica B 180, 468 (1992).

[10] M. Wagner and B.A. Wolf, Polymer 34, 1460 (1993).

[11] J.L. Adams, D.J. Quiram, W.W. Graessley, R.A. Register and G.R. Marchand, Macromolecules 31, 201 (1998).

[12] G. Gompper and M. Schick, Phys.Rev.Lett. 65, 1116 (1990).

[13] G. Gompper and M. Schick, Phys.Rev.E 49, 1478 (1994).

[14] B. Widom, Phys.Chem. 100, 242 (1996).

[15] P.K. Janert and M. Schick, Macromolecules 1, 137 (1997).

[16] L. Kielhorn and M. Muthukumar, J.Chem.Phys.107, 5588 (1997).

[17] D. Choy and S. Chen, Phys.Rev.E 61, 4148 (2000).

[18] H. Gang, A.H. Krall, H.Z. Cummins and D.A. Weitz, Phys.Rev.E 59, 715 (1999).

[19] E.Y. Sheu, S.H. Chen, J.S. Huang and J.C. Sung, Phys.Rev.A 39, 5867 (1989). 
[20] P. Sollich, F. Lequeux, P. Hébraud and M.E. Cates, Phys.Rev.Lett. 78, 2020 (1997); P. Sollich, Phys.Rev.E 58, 738 (1998).

[21] L.C.E. Struik, Physical aging in amorphous polymers and other materials (Elsevier, Amsterdam, 1978).

[22] J.-Ph. Bouchaud, L.F. Cugliandolo, J. Kurchan and M. Mézard, Spin glasses and random fields, Ed.: A.P. Young (World Scientific, Singapore, 1998).

[23] J.-L. Barrat and L. Berthier, Phys.Rev.E 63, 012503 (2001).

[24] R.G. Larson, Rheol.Acta 31, 497 (1992); The Structure and Rheology of Complex Fluids (Oxford University Press, 1999).

[25] X.L. Wu, D.J. Pine and P.K. Dixon, Phys.Rev.Lett. 66 , 2408 (1991).

[26] D. Roux, F. Nallet and O. Diat, Europhys.Lett. 24, 53 (1993).

[27] F. Boue and P. Lindner, Europhys.Lett. 25, 421 (1994).

[28] J. Yamamoto and H. Tanaka, Phys.Rev.Lett. 74, 932 (1995).

[29] S.-E. Phan, Z. Cheng, J. Zhu, P.M. Chaikin, J.H. Dunsmuir, R.H. Ottewill and W.B. Russel, Phys.Rev.E 54, 6633 (1996).

[30] K. Migler, C. Liu and D.J. Pine, Macromolecules 29, 1422 (1996).

[31] U. Batra, M. Pitsikalis, S. Sioula, J.W. Mays, J.S. Huang and W.B. Russel, Macromolecules 30, 6120 (1997).

[32] A. Léon, D. Bonn, J. Meunier, A. Al-Kahwaji, O. Greffier and H. Kellay, Phys.Rev.Lett. 84, 1335 (2000).

[33] G.H. Fredrickson, J.Chem.Phys. 85, 3556 (1986).

[34] L. Leibner, Macromolecules 13, 1602 (1980).

[35] M.E. Cates and S.T. Milner, Phys.Rev.Lett.62, 1856 (1989).

[36] K.A. Koppi, M. Tirrel, F.S. Bates, K. Almdal and R.H. Colby, J.Phys.(France) 2, 1941 (1992); K.A. Koppi, M. Tirrell and F.S. Bates, Phys.Rev. Lett. 70, 1449 (1993).

[37] N.P. Balsara, B. Hammouda, P.K. Kesani, S.V. Jonnalagadda and G.C. Straty, Macromolecules 27, 2566 (1994); N.P. Balsara and B. Hammouda, Phys.Rev.Lett. 72, 360 (1994).

[38] A.I. Nakatani, F.A. Morrison, F.A. Douglas, J.W. Mays, C.L. Jackson, M. Muthukumar and C.C. Han, J.Chem.Phys. 104, 1589 (1996).

[39] Chien-Yueh Huang and M. Muthukumar, J.Chem.Phys. 107, 5561 (1997).

[40] G. Pätzold and K. Dawson, Phys. Rev. E 54, 1669 (1996).

[41] F. Corberi, G. Gonnella and D. Suppa, Phys.Rev.E 63, 040501 (2000). 
[42] F. Drolet, P. Chen and J. Vinals, Macromolecules 25, 8603 (1999).

[43] M. Hennes and G. Gompper, Phys.Rev.E 54, 3811 (1996).

[44] P.C. Martin, E.D. Siggia and H.A. Rose, Phys.Rev.A 8, 423 (1973).

[45] R. Baush, H.K. Janssen and H. Wagner, Z.Phys.B 24, 113 (1976).

[46] C. De Dominicis and L. Peliti, Phys.Rev.B 18, 353 (1978).

[47] M. Teubner and R. Strey, J. Chem. Phys. 87, 3195 (1987).

[48] G.H. Fredrickson and F.S. Bates, J.Chem.Phys. 85, 633 (1986).

[49] A. Onuki and K. Kawasaki, Suppl.Prog.Theor.Phys. 64, 436 (1978); Ann.Phys. 121, 456 (1979); Phys.Lett. 72A, 233 (1979).

[50] K.Binder, J.Chem.Phys.79, 6387 (1983).

[51] R. Kubo, The Fluctuation Dissipation Theorem, Rep.Prog.Phys. 29 Part I, 255 (1966).

[52] R. Kubo, M. Toda and N. Hashitsume, Statistical Physics II, edited by P. Fulde (Springer, Berlin, 1991).

[53] J.H. Schulman,R. Matalon and M. Cohen, Discuss.Faraday Soc. 11, 117 (1951)

[54] D.J. Cebula, R.M. Ottewill, J. Ralston and P.N. Pusey, J.Chem.Soc. Faraday Trans. 1 77, 2585 (1981)

[55] L. Auvray, J.P. Cotton, R. Ober and C. Taupin, J.Phys.Chem 88, 4586 (1984); J.Phys. 45, 913 (1984).

[56] F. Lichterfeld, T. Schmeling and R. Strey, J.Phys.Chem. 90, 5762 (1986), and references therein.

[57] R.B. Stinchcombe, J.E. Santos and M.D. Grynberg, J.Phys.A 31, 541 (1998).

[58] G. Parisi, Statistical Field Theory, Ed.: Advanced Book Classic, Perseus (1998).

[59] G.H. Fredrickson, J.Chem.Phys. 85, 5306 (1986).

[60] J. Zinn-Justin, Quantum Field Theory and Critical Phenomena, Ed.: Clarendon Press (Oxford, 1989); I.M. Gel'fand and G.E. Shilov, Generalized Functions, Ed.: Academic Press (New York and London, 1964). 